\title{
Research on transformation mode of scientific and technological achievements with different transaction costs: taking the electric power industry as an example
}

\author{
Kaiyan Lei ${ }^{1, *}$ and Meimei Zhang ${ }^{1}$ \\ ${ }^{1}$ Department of economics and management, North China Electric Power University, Baoding \\ 071003, Hebei Province, China
}

\begin{abstract}
Technological innovation and achievement transformation are an important starting point for constructing an industrial innovation ecosystem and coping with hindered external demand. This article starts with the ecological chain of technological achievement transformation, constructs a transaction cost and benefit model of three transformation modes of direct transformation, intermediary participation, and coconstruction cooperation, and systematically analyses its transaction costs, benefits, application and the value space of the transaction subject of each mode. With the participation of intermediaries and investors, the "government, industry, university, research, and financial agency" achievement transformation service platform not only reduces transaction costs but also greatly increases returns. Using the advantages of big data and blockchain can obtain more added value than other models, bringing more opportunities for both supply and demand.
\end{abstract}

\section{Introduction}

In recent years, our country has intensified its efforts to reform its science and technology system, and comprehensively promoted the development of intermediaries and innovation centres for the transformation of technological achievements. But the annual conversion rate of achievements is roughly distributed between $10 \%$ and $15 \%$, which is much lower than that of developed countries in the world.

From the perspective of the various types of domestic innovation entities, Tsinghua University adopts the local research institute model with Shenzhen Tsinghua University Research Institute as a typical example [1]; the National University Science Park is an important part of the national innovation system [2].State Grid has established a production-university-research innovation system with State Grid Economic Research Institute, State Grid Energy Institute, and China Electric Power Research Institute. "China Power Valley" deeply integrates industry, market, technology research and development and talent education. However, due to factors such as the relatively fixed and single market

${ }^{*}$ Corresponding author: $\underline{\text { sxpylky@,126.com }}$ 
for power scientific and technological achievements and the relatively closed power system, there are problems such as difficulty in transforming value, disconnection between achievements and market demand, and unclear asset disposal rights, which result in the phenomenon that the conversion rate of achievements in the power industry is still low.

\section{Transaction cost analysis of the transformation of scientific and technological achievements}

In the transformation of achievements, the government provides policy support through benefit evaluation to realize the industrialization and marketization of scientific and technological achievements [3]. Enterprises and scientific research institutions and universities apply "secondary innovation" to the achievements, tapping the potential of a large number of deposited technologies in universities [4]. Under the guidance of value cocreation in the transformation process chain of scientific and technological achievements, each participant hopes to obtain more benefits within the range of cost tolerance.

\subsection{Transaction Cost of Direct Conversion Model-Revenue Model}

Here we consider the four costs that have the greatest impact on exogenous transaction costs: search costs $\left(C_{01}\right)$, negotiation costs $\left(C_{02}\right)$, decision-making costs $\left(C_{03}\right)$, and supervision costs $\left(C_{04}\right)$.Assuming that in the achievements' market, the achievements provider is the receiver of the price. The price $P$ and the market demand $q$ are exogenous variables, and the retention value of the achievements of the provider and the demander is $k$, $t$, and does not change with the market structure [5].

The basic cost of direct conversion between supply and demand parties is:

$$
C_{B}=\Sigma^{4}{ }_{i=1} C_{0 i}+C_{1}
$$

The transaction cost of the provider and the demander are:

$$
\begin{aligned}
& C_{T I}=m_{1}\left(\Sigma_{i=1}^{4} \partial_{i} C_{0 i}+C_{1}\right) \\
& C_{X I}=m_{2}\left(\Sigma_{i=1}^{4} \beta_{i} C_{0 i}+C_{l}\right)
\end{aligned}
$$

Among them, $\partial_{i}\left(0<\partial_{i}<1\right)$ and $\beta_{i}\left(0<\beta_{i}<1\right)$ are the elasticity coefficient of each item, and its value depends on the effort level of both parties to reduce the cost of each exogenous transaction; $m_{l}$ and $m_{2}$ are the cost coefficient of the provider and demander.

The net income of the provider and the demand side are:

$$
\begin{gathered}
W_{l}=P-t-C_{T l}+W^{\prime} \\
W_{l}=k-C_{X I}-P
\end{gathered}
$$

Among them, $W^{\prime}$ is the added value increment obtained after the achievement transaction, such as achievement application feedback, credibility, etc.

Obtained by the net income $W_{l} \geq 0$ and $W_{l} \geq 0$ :

$$
\begin{gathered}
\Sigma_{i=1}^{4} \partial_{i} C_{0 i}+C_{l} \leq C_{B} \leq\left(P-t+W^{\prime}\right) / m_{1}=C_{B 1} \\
\Sigma_{i=1}^{4} \beta_{i} C_{0 i}+C_{1} \leq C_{B} \leq(k-P) / m_{2}=C_{B 2}
\end{gathered}
$$

Therefore, the basic cost transaction range in the direct conversion model is: 


$$
C_{B} \leq \min \left\{C_{B 1}, C_{B 2}\right\}
$$

\subsection{Cost-benefit model of intermediary participation}

Intermediary agencies will separately accrue intermediary service fees $u_{1}, u_{2}\left(u_{1}>0, u_{2}>0\right)$ to both parties of the achievement transaction. The operating cost of the intermediary agency itself is $C_{M}$, and the participation efficiency is $\theta(0<\theta<1)$.

The transaction cost of the provider and the demander are:

$$
\begin{aligned}
& C_{T 2}=\theta m_{1}\left(\Sigma_{i=1}^{4} \partial_{i} C_{0 i}+C_{1}\right)+u_{1} \\
& C_{X 2}=\theta m_{2}\left(\Sigma^{4}{ }_{i=1} \beta_{i} C_{0 i}+C_{1}\right)+u_{2}
\end{aligned}
$$

The net income of the provider and the demand side are:

$$
\begin{aligned}
& W_{2}=P-t-C_{T 2}+W^{\prime} \\
& W_{2}{ }^{\prime}=k-C_{X 2}-P
\end{aligned}
$$

Obtained from the net income $W_{2} \geq 0$ and $W_{2}^{\prime} \geq 0$ :

$$
\begin{gathered}
\Sigma_{i=1}^{4} \partial_{i} C_{0 i}+C_{l} \leq C_{B} \leq\left(P-t-u_{l}+W^{\prime}\right) / m_{l} \theta=C_{l} \\
\Sigma_{i=1}^{4} \beta_{i} C_{0 i}+C_{l} \leq C_{B} \leq\left(k-u_{2}-P\right) / m_{2} \theta=C_{2}
\end{gathered}
$$

Therefore, the range of transaction costs in the transformation mode of scientific and technological achievements with the participation of intermediaries is:

$$
C_{B} \leq \min \left\{C_{B 1}, C_{B 2}\right\}
$$

The income increment $L$ of the provider with the participation of intermediaries is:

$$
L=H+C+L^{\prime}
$$

$H$ is the increase in income from unit achievement conversion when intermediary agencies participate; $C$ is the cost reduction of intermediary agency participation relative to direct conversion; $L^{\prime}$ is the added value increment obtained after the transaction.

The income increment $U$ of the demand side is:

$$
U=U(C)-U^{\prime}
$$

Among them, the income generated after the demander puts the technological achievements into use can be measured by the cost increment $C$ of the provider as the value increment represented by the variable $U(C) ; U^{\prime}$ is the cost increment of the demander.

\subsection{The profit distribution model of cooperative construction}

Based on the "principle of sharing investment risks and sharing benefits", each entity needs to consider the investment amount $I$, risk coefficient $\gamma$, income $R$, income distribution ratio $\varphi$ and contribution coefficient $j$.

If the number of cooperative entities in the process of transforming scientific and technological achievements is $n$, consider the income distribution ratio $\varphi_{i}$ of the $i_{t h}$ cooperative entity as a function of $I_{i}$ and $\gamma_{i}$, that is, $\varphi_{i}\left(I_{i}, \gamma_{i}\right), i=1,2 \ldots n$. The return of the 
cooperative entity increases with the increase in risk and investment. The income from the transformation of achievements is shared by all partners, that is, $\Sigma^{n}{ }_{i=1} \varphi_{i}=1$.

The contribution and income of each subject need to match:

$$
R_{1} / j_{1}=R_{2} / j_{2}=\ldots R_{n} / j_{n}
$$

In the process of transforming scientific and technological achievements, each cooperative entity should be profitable, and the benefits of each entity are:

$$
R_{i}=\left(I_{i} \gamma_{i} / \Sigma^{n}{ }_{i=1} I_{i} \gamma_{i}\right) j_{i} R, \quad i=1,2, \ldots n
$$

\section{Analysis of new energy technology achievement transformation mode based on information technology}

Energy data has huge potential application value in promoting the on-demand flow of energy resources and the optimization and transformation of energy structure [6]. The new power infrastructure is a propeller for the transformation and upgrading of the power industry, which will derive new market demands for the transformation of technological achievements. Combining the industrial characteristics and transaction costs of this field, establish a new energy technology transformation model based on information technology.

\subsection{Direct conversion and industry-university-research cooperation model}

$C_{01} \sim C_{04}$ of direct conversion are relatively large. Subjects with channel advantages, industrial advantages, professional advantages are suitable for direct conversion models. The supply and demand parties realize the transformation through direct forms such as project cooperation, patent transfer, technical services, etc. The benefits obtained are the value of the achievements themselves and the added value increment $W^{\prime}$.

Enterprises and university research institutions jointly funded to build R\&D platforms. The achievements of the research centre under this model are directly connected to the enterprise, greatly reducing $C_{01}$ and $C_{03}$. The two parties distribute the added value $R$ brought by the application of the achievements according to the proportion $\varphi$, and jointly share R\&D risks. For example, the U.S. Energy Innovation Centre plays a role in linking basic research and technology applications [7], adopting the model of "leadership duality, structure duality, and scenario duality".

\subsection{In-depth collaboration model of "government, industry, university, research, finance and agency"}

From the perspective of the transaction cost, because the actual intermediary fee $u$ is less than the reduction of $C_{01} \sim C_{04}$, the transaction cost of both parties decreases. At the same time, due to the time-sensitive services of intermediary agencies, services such as credit feedback and cooperative evaluation have relatively increased the added value of both parties, such as credibility, cooperation potential, etc., and ultimately enabled both the supply and demand parties to obtain increased revenue $L$ and $U$. Therefore, scientific and technological achievements with a broad market can increase the main body's income through the participation of intermediary agencies.

In this model, intermediary service agencies provide comprehensive services such as information collection, technical evaluation, market research, development operations research, etc., which can help both the supply and demand parties reduce economic costs and promote the "secondary innovation" of the results of both parties [8]. It should be 
focused on. Build a new energy power technological achievements transformation service platform, set up a technology comprehensive service company or technology transformation service office, etc., and it integrate all parts of the "government, industry, university, research, and finance". Regularly release and update a large number of scientific and technological achievements related to scientific research institutes and enterprise applications. Add financing section, shared resources section and conversion trading section. Provide chain services such as scientific research team information, enterprise demand information, legal and regulatory consultation, technical achievement information, and transaction contract signing to ensure that all kinds of users collect resources and information related to the innovation main body of achievement transformation in the shortest time. Intermediary service agencies can provide professional and financial two-way guarantees for the transformation of scientific research results through shareholding, equity participation, co-investment and low-interest loans. Intermediary agencies provide part of the source of funds and obtaining reasonable income. Tax incentive policies play an important role in promoting the transformation of scientific and technological achievements of Chinese universities. Drawing lessons from the British and American "neutralization" tax system design concepts, build a unified tax policy system under different modes of transformation of scientific and technological achievements [9].

\subsection{Online and offline collaboration mode based on big data and blockchain}

It refers to the establishment of a new energy power full ecological chain scientific and technological achievements operation platform, scientific evaluation of technological achievements, and the use of blockchain technology for tracking or tracing. Chinese universities and large state-owned enterprises occupy a core position in the invention patent cooperation network [10]. Big data technologies such as network graphs can be used to obtain new achievements in a timely manner, and to establish a patent database, intellectual property database and technological innovation achievement database in the new energy power industry; The use of big data technology can build a fast identification, accurate matching docking transaction mechanism and a security management mechanism based on the principle of data desensitization. It conducts diversified data analysis on the technical requirements, corporate assets and development prospects released by the technical demander, as well as the technical achievements, technical strength, number of patents and other aspects of the technical provider. Docking rules will be formulated to achieve the needs of the main body of the technology transformation ecological chain and the precise matching of achievements information. At the same time, it carries out the task of forecasting the supply and demand of new energy power scientific and technological achievements, guiding the technology provider to determine the research topic, and actively pushing relevant information about transformable achievements to the technical demand side; Establish an online value evaluation channel for new energy power scientific and technological achievements, carrying out intellectual property analysis and review. Through the traceability and anti-counterfeiting of blockchain technology and the smart contract technology that standardizes the process, it provides technical support for patent mining and transaction and determination of property rights. Entrusted agents for offline achievement transformation, technical achievement observation meetings, etc., promoting offline supplydemand matching, technical consultation and transaction negotiation.

This model forms an open and shared innovation achievement transformation ecosystem, breaking the data islands among technology holders, technology transformation parties, and technology transfer institutions. It eliminates the problem of information asymmetry in the process of technological transformation, thereby reducing the search cost $C_{01}$. It solves the problems of the right to confirm innovation results and transaction trust, thereby reducing 
decision-making costs $C_{03}$. Big data and blockchain technology have the characteristics of high timeliness, irreversibility, and low value density, which accelerate the transformation. The amount of added value obtained by both parties to the transaction is much greater than other models, thereby bringing more opportunities for both parties.

\section{Conclusion}

This article mainly proposes a new energy power technology achievement transformation model based on information technology from the perspective of transaction cost-income. The main conclusions are as follows:

First, achievements transformation is divided into direct and indirect transformation, third-party transformation, and establish their transaction cost-benefit model.

Second, the direct conversion model has high transaction costs and is suitable for entities with industrial and professional advantages. The joint construction of industryuniversity-research cooperation platform model can greatly reduce search costs and decision-making costs, and is suitable for enterprises with large new energy power industries.

Third, the "Politics, Industry, University, Research, Financial and Intermediary" achievement transformation service platform, thanks to the participation of intermediaries and investors, not only reduces transaction costs but also greatly increases returns. Using the advantages of big data and blockchain can obtain more added value than other models, and bring more opportunities for both supply and demand.

Project Name: New energy big data operation platform under the ubiquitous power Internet of Things. Item Number: 202010054105. Fund projects: *Hebei Province's soft science project "Research on Digital Agriculture with Double Helix Structure-New Kinetic Energy for Rural Revitalization" (20557652D).

\section{References}

1. Xinghua Xie, Zhihong Zi. S. T. Man. Res. J. 24, 109-114(2018)

2. Miaojun Dong, Zhen Lu, etc. Science and Technology of Chinese Universities. J. 10, 82-85(2019)

3. Jiashu Liu, Suga Lirong. Science of Science and Management of Science and Technology. J. 6, 33-40(2011)

4. Hu Zhang, Liu Yang, Wei He. Scientific Research Management. J. 38(01), 676679(2017)

5. Meimei Zhang. Industrial Technology Economy. J. 06, 72-74(2008)

6. Yuanyuan Wang, Hongkun Bai, etc. Smart Electric Power. J. 48(03), 15-21+29(2020)

7. Lin Wen, Guanghua Chen. Technical economy. J. 35(10), 59-65(2016)

8. Jie Dong, Zugong Chen. Scientific Management Research. J. 05, 44-46(2009)

9. Xuhua Chang, etc. Research in Science of Science. J. 36(04), 635-643(2018)

10. Yong Yang, Luhan Wang. Studies in Science of Science. J. 38(07), 1227$1235+1316(2020)$ 